\title{
Development of an Universial Allergen Sensor System
}

\author{
A.Goehlich, K.Burmester, Y.Celik, R.Klieber, D.Greifendorf, N.Haas, H.Vogt \\ Fraunhofer Institut für Mikroelektronische Schaltungen und Systeme, \\ Finkenstraße 61, 47057 Duisburg, andreas.goehlich@ims.fraunhofer.de
}

\begin{abstract}
:
In this contribution we report on results of the Euregio funded project "Unihealth" that is dedicated to the development of a micromechanical sensor intended for the detection of important allergens.

The "Unihealth" project aims at the development of a cost effective lable free bio sensor system that is intended for point of care applications concerning the detection of a large range of allergens e.g. the papain enzyme, gluten or allergens causing the peanut-allergy. The adopted sensor principle relies on electro statically driven resonating micromechanical membrane structures with a functionalized surface that allows for the selective binding of allergens.
\end{abstract}

Key words: allergen, MEMS, CMOS-integrated sensor, membrane, nonlinear oscillator

\section{Introduction and Motivation}

The need for cost effective allergen monitoring systems in POC applications is of crucial importance due to the increasing number of people suffering from different food allergies caused e.g. by peanuts, milk, eggs or soya. Up to about $30 \%$ of the European population claim to be are affected by food allergies [1]. About 20000 different substances are suspected to cause allergies.

Within the framework of the „Unihealth“ project a simple and cost-effective allergen detection system is developed utilizing a micromechanical bio-sensor intended for an everyday use application or for applications in fundamental research as well as for medical surgeries in the area of allergies.

Common biochemical analysis methods of allergens rely on antibody based techniques as ELISA (enzyme linked immunoassay), DNA amplification methods as PCR or on analytical mass spectrometry and often require bulky laboratory analysis equipment, are time consuming and are with limited benefit for day to day home application.

Since the typical mass of the allergens is in the range of $10 \mathrm{kDa}$ to $80 \mathrm{kDa}$ the mass sensing with micro or nano structures is a promising detection principle. The current sensor system is based on the direct physical mass sensing with a micromechanical membrane in combination with a functionalized surface and allows the quantitative measurement of the analytes concentration. The functionalized surface layer is selective to certain target allergens according to the lock-and-key principle. An universal biochemical functionalization platform has been developed by the project partners (universities Wageningen and Nijmegen and by Surfix B.V.).

The utilization of functionalized oscillating micromechanical structures as film bulk acoustic wave resonators (FBARs) [2], cantilevers [3] and membrane structures [4]-[6] for mass sensing is well known from previous work of different groups.

The resonant mass sensing principle relies on the measurement of the frequency shift that is connected to an accumulated mass on an oscillating structure in analogy to the well known quartz micro balances. The measureable frequency shift is related to the mass change by the following relation that can be obtained by differentiation of the well known expression for the resonance frequency of the harmonic oscillator:

$$
\Delta f \cong-\frac{1}{2} \frac{\Delta m}{m} f_{0}
$$


Since the mass $\mathrm{m}$ of a microstructure can be made quite small very sensitive structures can be realized with methods of micro or nano patterning.

Typically a self oscillating electronic read out circuit is set up and the oscillation frequency of the device is measured by frequency counting. In the group of Khuri-Yakub CMUT (capacitive micro mechanical ultrasonic transducer) structures have been successfully applied for sensing of gaseous analytes [4]. In principle these structures are build up as capacitive parallel plate structures with two electrodes that enclose an evacuated cavity.

In contrast to the very sensitive cantilever structures sealed membrane structures exhibit at the expense of sensitivity the inherent advantage of a higher mechanical robustness.

In this work we report on membrane structures that have been realized with a CMOS compatible pressure sensor technology. In the following section the sensor system and technology is explained.

\section{Sensor System and Technology}

The mass sensing element itself essentially consists of a circular capacitor comprising a flexible poly silicon membrane (with a thickness of approx. $1 \mu \mathrm{m}$ ) and a fixed high dose implanted counter electrode [Fig. 1].

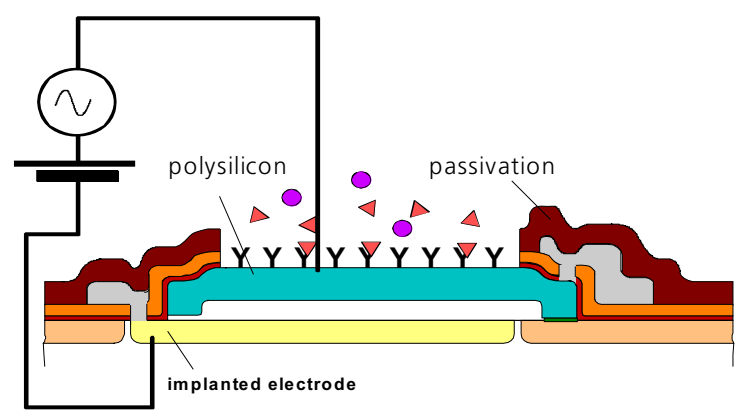

Fig. 1. Schematic presentation of the sensor cell.

The cavity between the electrodes is created with the aid of a sacrificial oxide that is removed by a $50 \% \mathrm{HF}$ solution. The cavity is hermetically sealed at low pressure with a LPCVD deposited silicon nitride layer. This robust nitride sealing ensures the long term stability of the device. Moreover the sealed structure ensures the operation also after loading the sensor surface with a fluid without the danger of a mechanical sticking by adhesion forces. The low pressure inside the cavity considerably reduces the damping effect at atmospheric pressure.
The basic idea of the sensor system is to load the surface with the solution containing the analyte (e.g. allergens). After a certain reaction time the analyte binds with the functionalized surface and generates the mass increase. Subsequently the surface is washed to remove all unbound residues and dried. The mass increase due to the target analyte is detected either by a pre-post-measurement or alternatively by a simultanuous measurement of a non functionalized reference element (see also modeling section).

The sensor element can be monolithically integrated with a read out circuitry that is realized with a $1.2 \mu \mathrm{m}$ CMOS production process. Stand alone devices with pad connections only as well as devices with a monolithically integrated band pass filter, a programmable amplifier stage, a peak-to-peak sampling stage including low pass filtering have been realized. A photograph of the ASIC (application specific integrated circuit) and a circuit bloc diagrammatic representation is depicted in figure $2 \mathrm{a}$ ) and b).

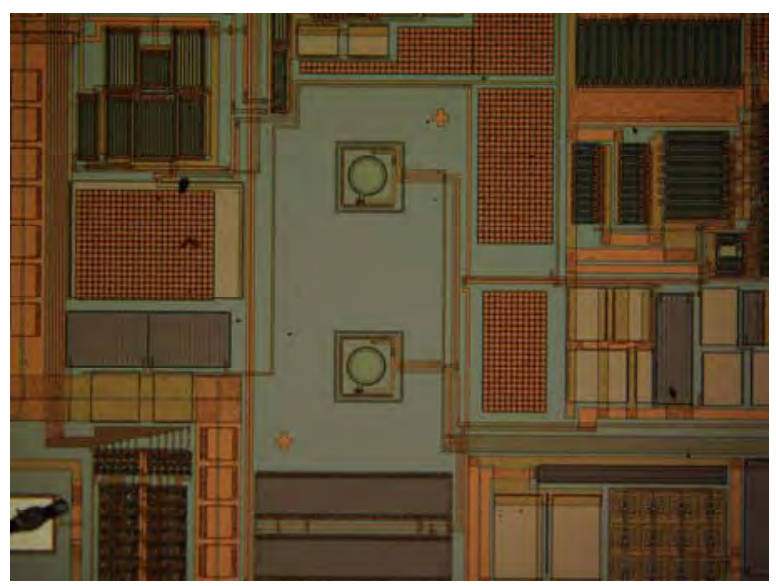

Fig. 2a) Photograph of the integrated sensor ASIC.

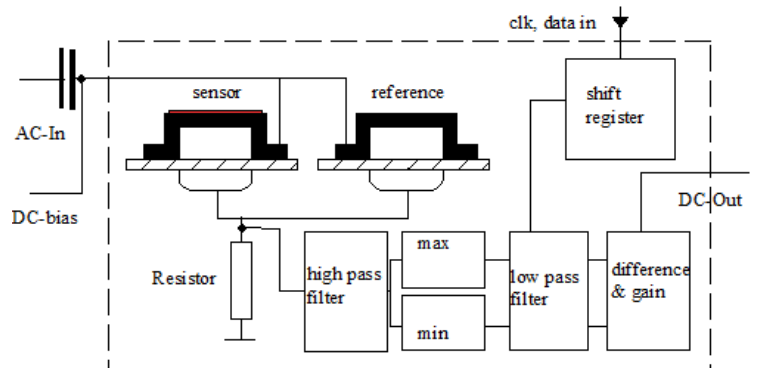

Fig. 2b) Sche $\overline{\text { matic }} \overline{\text { bloc }} \overline{\text { diagram }} \overline{\text { of }} \overline{\text { integrated }} \overline{\mathrm{J}}$ ASIC. In the sketched version a "double structure" composed of a sensor and reference element is utilized.

The integrated ASIC is excited with an external AC-signal in the $\mathrm{MHz}$ range and a DC-bias in the range $0-30 \mathrm{~V}$. The excitation signal is generated by an external frequency generator. 
The integrated ASIC delivers an analogous output signal in the range $0-5 \mathrm{~V}$, that is proportional to the peak-to-peak value of the oscillation. The resonance spectrum is determined by tuning the excitation frequency across the resonance. The DC-bias of the excitation is adjusted in a way that the membrane is operated in a non linear regime taking advantage from a sharp transition observed in the resonance feature of the spectral profile (see modeling section below). Versions of the ASIC with a double sensor structure i.e. a combination of a sensing element and a non functionalized reference element have been also been realized ("double structure"). In this case the currents of bothelements are added in a resistor and the superposition of the spectra of both membranes is detected. This is explained in more detail in the modelling section.

\section{Modelling of the Sensor Structure}

The basic features as the expected resonance frequency and the expected sensitivity (see Figure 3) of the sensor element are modeled by finite element modeling (FEM) with the commercial software package COMSOL. The specific axial geometry of the sensor element and the material properties of the layer stack comprised of poly silicon and the sealing nitride have been taken into account. In order to estimate the sensitivity two limiting scenarios have been considered for the accumulated mass: the maximum sensitivity is obtained if the mass of the target analyte is concentrated in the center of the membrane, the alternative scenario is the uniform coverage with the analytes mass.

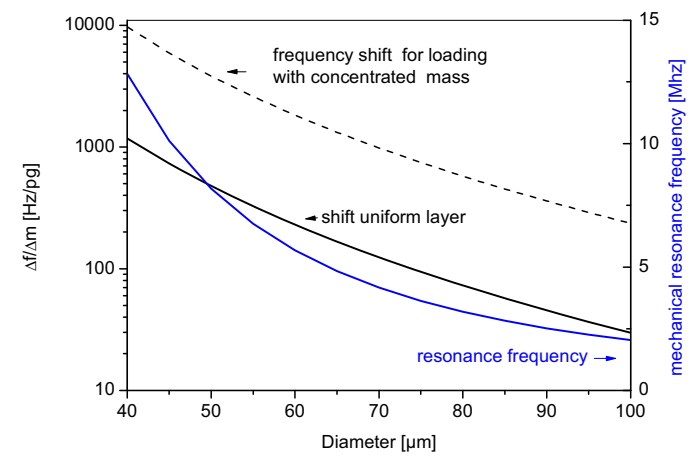

Fig.3 FEM simulation of frequency and sensitivity for different membrane diameter (layout). Left axis calculated shift, right axis resonance frequency.
In order to simulate the dynamical behaviour of the electrostatically driven membranes also for large deflections a simple 1d-approach has been utilized here.

We have adopted a simple oscillator type differential equation for the modelling of the membrane motion and for the the electrical current $[7,10]$. The deflection $w(r, t)$ of the membrane is approximated by the product of a time dependent amplitude $\mathrm{w}_{0}(\mathrm{t})$ and a time independent axisymmetric spatial deflection profile $f(r)$, i.e.: $w(r, t)=w_{0}(t) f(r)$ [7]. The driving bias $U(t)$ is composed of an AC- and a DCvoltage: $U(\mathrm{t})=\mathrm{U}_{A C}+\mathrm{U}_{\mathrm{DC}} \sin (\Omega \mathrm{t})$. $\Omega$ denotes the excitation frequency.

The motional equation is then approximated by eq. 2 :

$$
\begin{gathered}
m_{\text {eff }} \frac{d^{2}}{d t^{2}} w_{0}+\lambda \frac{d}{d t} w_{0}+k_{\text {eff }} w_{0}+k_{N L} w_{0}{ }^{3}=F_{e l}\left(t, w_{0}\right) \\
F_{e l}\left(w_{0}(t)\right)=\frac{\varepsilon \varepsilon_{0}}{2} U^{2}(t) \int_{0}^{R} \frac{2 \pi r d r}{\left(s p-w_{0}(t) f(r)\right)^{2}}
\end{gathered}
$$

$m_{\text {eff, }}, k_{\text {eff }}$ and $\lambda$ denote the effective mass, spring constant and the damping factor that is connected to the quality factor $Q$. $R$ is the effective radius of the membrane and $s p$ denotes the effective spacing between the electrodes. The value of the effective mass and spring constant are chosen to match the resonance frequency and stiffness of a plate with radius $R$ und thickness $t$. The coefficient $k_{n l}$ accounts for the non linear mechanical spring response of the membrane obtained for large deflections. These parameters have to be considered as lumped element parameters that can to be fitted in principle to experimental results. $F_{e l}$ is the non linear driving force due to the electrostatic pressure inside the cavity. The other parameters have their usual meaning. The non linear driving force $F_{\mathrm{el}}\left(\mathrm{w}_{0}(\mathrm{t})\right)$ can be expanded in powers of the amplitude $w_{0}$ and additional anharmonic terms -besides the mechanical nonlinearity- can be obtained. Since these terms can have different algebraic sign positive and negative anharmonic terms are possible. The spatial dependence of the deflection profile is approximated by a fixed profile:

$$
f(r)=\left(1-\frac{r^{2}}{R^{2}}\right)^{n}
$$

The empirical parameter $\mathrm{n}$ allows to approximate the shape of the surface e.g. a flat surface $(n=0)$, a parabola type surface $(n=1)$ or a clamped plate type surface $(n=2)$. From interferometric measurements it was found that 
our membranes may be described by a value of $\mathrm{n}$ close to 1.

With the known deflection amplitude $w_{0}(t)$ the electrical current $\mathrm{I}(\mathrm{t})$ that flows through the device is derived by :

$I(t)=\frac{d}{d t}[C(t) U(t)]=\frac{d}{d t}\left[U(t) \varepsilon \varepsilon_{0} \int_{0}^{R} \frac{2 \pi r d r}{\left(s p-w_{0}(t) f(r)\right)}\right]$

The differential equation (2) has been solved numerically with the aid of the Runge-Kutta method. The electrical current has been deduced from eq. 4 using analytical solutions for the integrals contained in eq. $2 b$ and eq. 4 . The developed software then calculates the amplitude and the phase of the mechanical motion. The electrical current is obtained from the time dependent solution of eq. $2 a$ and eq. 4 . Due to the frequency doubling effect by the non linear excitation $\propto\left(\mathrm{U}_{\mathrm{DC}}+\mathrm{U}_{\mathrm{AC}}(\mathrm{t})\right)^{2}$ two peaks in the spectrum are generated, one peak near $\Omega=\omega_{0} / 2$ arising from the contribution $\propto \mathrm{U}_{\mathrm{AC}}(\mathrm{t})^{2}$ and the other peak near $\Omega=\omega_{0}$, with $\omega_{0}$ denoting the mechanical resonance frequency. The peak near $\Omega=\omega_{0}$ is generated through the mixed term of the excitation force proportional to $U_{D C} U_{A C}$ $\sin (\Omega \mathrm{t})$ and is commonly used in the mass sensing application.

Typical frequency spectra calculated with the lumped element model are shown in figure 4 (deflection amplitude) and figure 5 (current amplitude) for different DC-bias with a fixed ACamplitude $U_{A C}=1 \mathrm{~V}$. The underlying simulation parameters are indicated in the graphs. Some essential features of the sensor elements behavior can be deduced from figure 4 . Firstly in the deflection amplitude spectrum the two resonant features near $\omega_{0} / 2$ and $\omega_{0}$ are observed as outlined above.

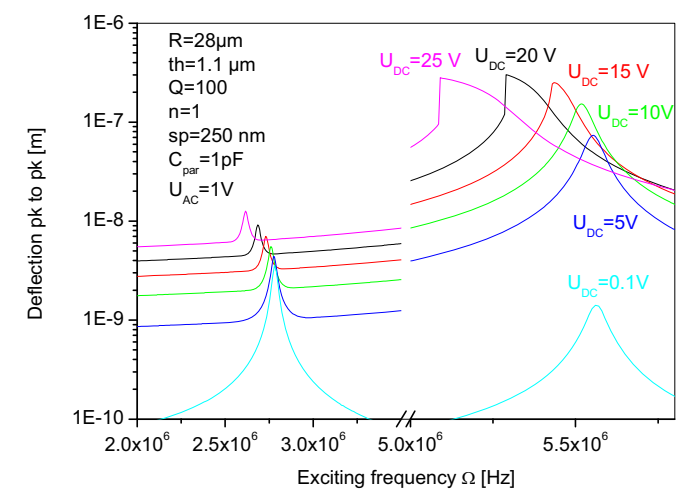

Fig. 4. Simulated amplitude spectra of the mechanical deflection for different DC-bias.
Secondly it is observed that with increasing DCbias the resonant features shift to lower frequency. This is well known as the so called spring softening effect that is related to the distortion of the effective potential by the increasing external bias. In addition the spectral profiles exhibit an asymmetric shape and a steep edge with increasing DC-bias due to the anharmonicity of the effective potential. Similar findings have been also reported in refs. [8-10]. The amplitude spectrum of the electrical current exhibits a resonant behavior only at $\Omega=\omega_{0}$. No resonant peak in the amplitude spectrum is observed near $\Omega=\omega_{0} / 2$ in the amplitude spectrum (but is observed in the phase spectrum-not shown).

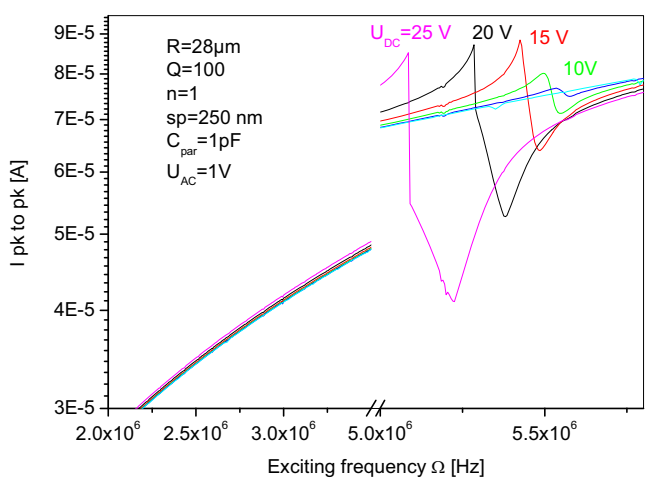

Fig. 5. Simulated amplitude spectra of the electrical current for different $D C$ - bias.

For smaller values of the DC bias the resonant feature exhibits the typical smooth resonance feature that can be described by the Butterworth von Dyke circuit (see e.g. the curve for $U_{D C}=10 \mathrm{~V}$ in Fig. 5). Due to the fact that the resulting impedance is composed of a time varying part (due to the oscillation) and static part in parallel the resonance feature exhibits a series and a parallel resonance.

In order to simulate the superposition spectrum of a "double structure" sensor the time resolved currents of the sensor element (loaded with a mass $\Delta \mathrm{m}$ ) and of the unloaded reference element are numerically added and the maximum amplitude of the composed current is determined as a function of the exciting frequency. As an example the resulting resonant features for a loading with $50 \mathrm{pg}$ and $100 \mathrm{pg}$ are depicted in figure 6 . In addition the differential spectrum is plotted. The spectral difference of the needle like peaks in the differential spectrum provides a very precise measure of the added mass. 


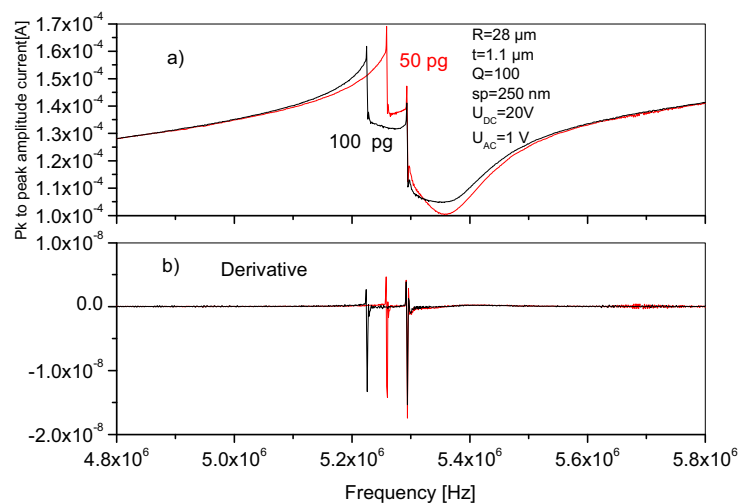

Fig. 6. Simulated superposition spectrum of a "double structure" with one membrane loaded with an additional mass. In figure b.) the differential spectrum is depicted.

As a consequence of the anharmonicity the spectrum exhibits also a hysteresis. Therefore the frequency has been tuned only in one direction e.g. from low to high frequencies.

\section{Experimental Characterization}

In figure 7a) a repetitive measurement (5 measurements) of the output signal of the integrated ASIC (with one sensor element) is depicted.

The excitation signal has been generated by an external frequency generator and the output voltage has been converted by an analog to digital converter. By raising the DC bias to $30 \mathrm{~V}$ the spectrum has been adjusted to exhibit the sharp transition mentioned above.

The spectra show a good reproducibility in particular for the spectral position of the sharp transition. For comparison also the lumped element simulation for the same excitation parameters $U_{A C}$ and $U_{D C}$ but manually "tuned" parameters $\mathrm{C}_{\mathrm{par}}$ (parasitic capacitance in parallel to static capacitance of the membrane), $\mathrm{sp}$ and $\mathrm{R}$ is depicted (fig. $7 \mathrm{~b}$ ). The simulation shows at least qualitatively the same shape of the resonant feature.

The sensitivity of the membranes (in this case investigated membranes with a diameter of 96 $\mu \mathrm{m})$ has been investigated experimentally by depositing platinum humps of different size by focused ion beam (FIB) deposition near the center of the membrane (see figure 8).

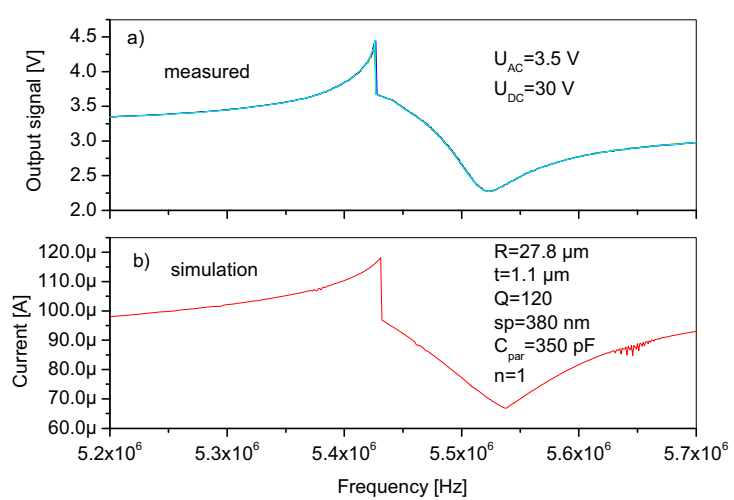

Fig. 7. Comparison of measured a) and simulated amplitude spectrum b) of the electrical current.

The mass of the deposited humps has been estimated from the optically measured volume and the effective mass density of the FIB deposited material [11]. With this "rough" calibration a value of about $320 \mathrm{~Hz} / \mathrm{pg}$ is obtained for membrane with diameter $96 \mu \mathrm{m}$ similar to value obtained by the FEM simulation for central load.

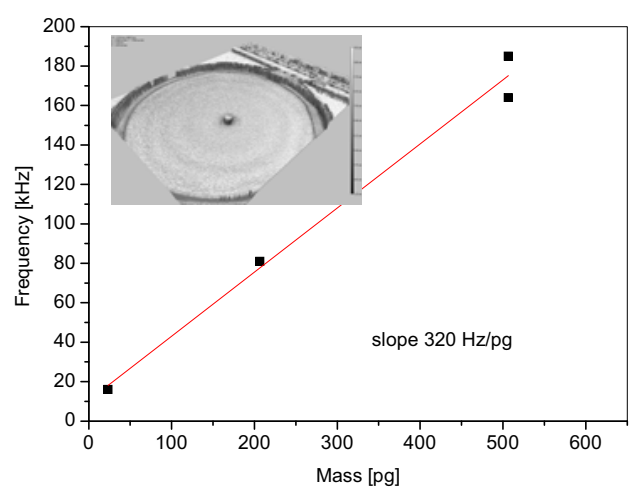

Fig. 8. A "rough" calibration of a sensor with diameter $96 \mu \mathrm{m}$ membrane. The topography of the membrane with a FIB deposited platinum hump is shown in the insert.

A biological attachment experiment of streptavidin with a biotin functionalized surface for the proof of principle has been conducted. The biotin surface preparation has been performed by Surfix B.V. and University of Wageningen. Details of these experiments will be published elsewhere. A preliminary measurement result is shown in figure 9. The frequency of the sharp transition in the resonant feature has been measured continuously before and after the loading with streptavidin and washing with deionized water (manually dispensed). 


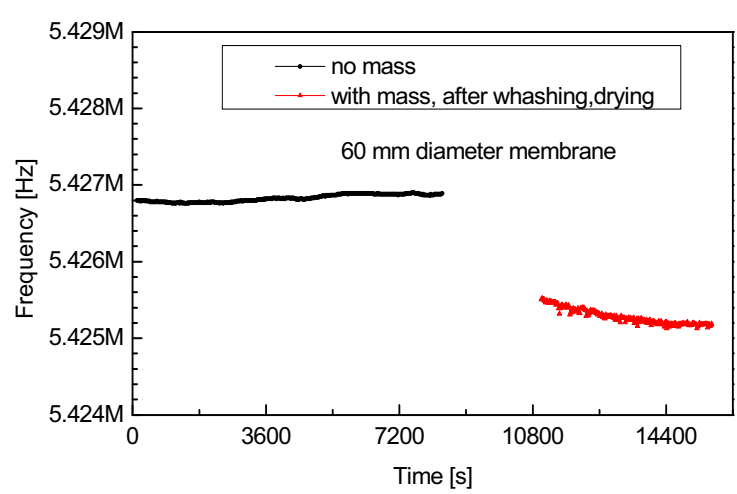

Fig. 9. Continuous measurement of the frequency position of the sharp transition before and after the streptavidin loading, washing and drying sequence.

A shift of about $1.7 \mathrm{kHz}$ is observed indicating an attachment of the bio molecules to the surface. This shift is within a factor of two in agreement with the assumption of a monolayer coverage of the membrane. The corresponding experiment with the loading with the buffer solution only shows only a negligible shift in contrast. Further validation experiments have to be performed however.

\section{Summary}

Within the „Unihealth“ project an allergen detecting system on basis of an electro statically excited polysilicon membrane is developed. The sensor elements were excited by a sufficient high DC-bias that generates a steep edge in the resonance profile that is utilized as a precise frequency marker. The spectra were measured by scanning the exciting frequency across the resonance. Also "double sensor elements" with a reference element have been realized enabling an in situ frequency reference. An ASIC with read out circuitry has been developed and characterized.

The shape of the resonance profile can be described by a 1d-numerical lumped element model. As a proof of principle a biological attachment experiment with the binding of streptavidin to a biotinalized surface has been conducted. Further experiments with allergen attachment are under study.

\section{Acknowledgement}

This work is co-financed by the INTERREG IV A Germany-Netherlands program through the EU funding from the European Regional Development Fund (ERDF), the Ministry for Economic Affairs, Energy, Building, Housing and Transport of the State of North-Rhine
Westphalia and the Dutch Ministry of Economic Affairs and the Province of Gelderland. It is accompanied by the program management Euregio Rhein-Waal.

\section{References}

[1] C.Mills, R.v.Ree, H.Breiteneder: Food allergy and intolerance in Europe - Future directions within the ERA. Proc.ICC Conference 2002 "Implementation of the European research area." p.95-96, 2002.

[2] H. Zhang, E. S. Kim.: Micromachined Acoustic Resonant Mass Sensor. J. Micromechanical Systems, Vol. 14, No.4, Aug. 2005, pp.699-706

[3] J. Verd, G. Abadal, M,V. Gaudò, A. Uranga, X. Borrisé, F. Campabadal, J. Esteve, E. F. Costa, F. Pérez-Murano: Design, Fabrication and Characterization of a Submicroelectromechanical Resonator with Monolithically Integrated CMOS Readout Circuit, J. Micromechanical Systems, Vol. 14, No.3, June 2005, pp. 508-518

[4] K. K. Park, H. J. Lee, G. G. Yaralioglu, A. S. Ergun, Ö. Oralkan, M. Kupnik, C. F. Quate, B. T. Khuri.Yakub: Capacitive micromachined ultrasonic transducers for chemical detection in nitrogen, Applied Physics Letters 91, 2007, 094102

[5] J. Lee, P. K. Kyu, Ö. Oralkan, M. Kupnik, B. T. Khuri Yakub: CMUT as a chemical sensor for DMMP detection", 2008, IEEE International Frequency Control Symposium, pp.434-439, 2008

[6] K. K. Park, H. J. Lee, Ö. Oralkan, M. Kupnik, B. T. Khuri-Yakub: Fabricating Capacitive Micromashined Ultrasonic Transducers via Local Oxidation and direct Wafer-Bonding. J. Micromechanical Systems, Vol. 20, No.4, Feb. 2011, pp.95-103

[7] A. Lohfink, P.-C. Eccardt, W. Benecke, H. Meixner: Derivation of a 1D CMUT Model from FEM Results for linear and nonlinear equivalent circuit, 2003 IEEE Ultrasonics Symposium, pp 465-468

[8] C. Gui, R. Legtenberg, H.A.C. Tilmans, J.H.J. Fluitman, M. Elwenspoek.: Nonlinearity and Hysteresis of Resonant Strain Gauges, J. Micromechanical Systems, Vol. 7, No.1, March 1998, pp.122-126

[9] R. M. C. Mestrom, R. H. B. Fey, K. L. Phan, H Nijmeijer: Simulations and experiments of hardening and softening resonances in a clamped-clamped beam MEMS resonator, Sensors and Actuators $A$ 162 (2010) 225-334

[10] M. I. Younis, F. Alasaleem: "Exploration of New Concepts in Mass Detection in Electrostatically Actuated Structures Based on Nonlinear Phenomena. J. Computational and Nonlinear Dynamics, Vol.4, April 2009, 021010-1-021010-14

[11] R. Abdolvand, Zhili Hao, F.Ayazi: A Temperature compensated $\mathrm{ZnO}$-on-Diamond Resonant Mass Sensor. IEEE SENSORS 2006, EXCO, Daegu, Korea / October 22-25, 2006 